\title{
Cognitive Behavioral Therapy for Management of Dyspnea: A Pilot Study
}

\author{
Marie T Williams PhD, Paul Cafarella, Catherine Paquet PhD, and Peter Frith MBBS
}

BACKGROUND: In patients with COPD, psychological interventions usually target generalized anxiety and depression rather than the sensation of breathlessness. The objectives of this pilot study were to develop and implement a cognitive behavioral therapy (CBT) program specific to the perceptual experience of breathlessness, identify practical issues in the study protocol, and estimate beneficial effects of combining the CBT program with comprehensive pulmonary rehabilitation. METHODS: The CBT program for the sensation of breathlessness (Breathing: Recognize sensations, Explore thoughts and beliefs, Validate thoughts as useful or harmful, Evolve and change behavior [BREVE]) was developed as a sequential series of 8 modules enabling it to be embedded within an 8-week comprehensive pulmonary rehabilitation program. When appropriate, outcomes from the pilot group (comprehensive pulmonary rehabilitation program + BREVE) were compared with those from a retrospective control group (comprehensive pulmonary rehabilitation program only). Outcomes included feedback provided by pilot study subjects, sensation of breathlessness (volunteered and endorsed descriptors of breathlessness), 6-min walk distance (6MWD), and St George Respiratory Questionnaire (SGRQ) total score. Within-group analyses were undertaken for descriptors of breathlessness (the McNemar test), whereas between-group analyses (repeated-measures analysis of variance, effect-size comparison) were conducted for the 6MWD and SGRQ total score. RESULTS: Pilot $(n=11)$ and control $(n=58)$ groups were not significantly different at baseline. Feedback indicated that the program structure and content were positively received. No significant changes were evident for the sensation of breathlessness or the SGRQ score ( $<4$ points). The 6MWD improved significantly in both groups, with the pilot group demonstrating greater gains compared with the control group (mean change of $57 \mathrm{~m}$ and effect size of $0.73 \mathrm{vs}$ mean change of $27 \mathrm{~m}$ and effect size of 0.23 ; between groups, $P=.03$, effect size of 0.69 ). CONCLUSION: The CBT program for the perceptual experience of breathlessness was feasible and well accepted by subjects, although the protocol raised a number of methodological limitations warranting modification. A larger randomized controlled trial is needed to determine the effectiveness and longerterm outcomes. Key words: breathlessness; chronic obstructive pulmonary disease; cognitive behavioral therapy; exercise capacity; psychological intervention. [Respir Care 2015;60(9):1303-1313. (C) 2015 Daedalus Enterprises]

\section{Introduction}

Dyspnea is a multidimensional perceptual experience that reflects an individual's awareness of breathlessness
Dr Williams is affiliated with the School of Population Health and Alliance for Research in Exercise, Nutrition and Activity (ARENA), School of Health Sciences, University of South Australia, Adelaide, South Australia, Australia. Mr Cafarella is affiliated with the Department of Respiratory Medicine, and Dr Frith is affiliated with the Southern Adelaide Health Service, Repatriation General Hospital, Daw Park, South intensity, sensory quality, and unpleasantness. ${ }^{1}$ Interventions that target the central processes for perception or cognition associated with dyspnea have been recognized as potential therapeutic strategies. ${ }^{2-4}$ To date, controlled trials of psychological interventions (including cognitive

Australia, Australia. Dr Paquet is affiliated with the School of Population Health, Spatial Epidemiology and Evaluation Research Group, Sansom Institute for Health Research, University of South Australia, Adelaide, South Australia, Australia. 
behavioral therapy [CBT]) in subjects with COPD have targeted generalized anxiety, panic, and depression rather than specifically focusing on the sensation of breathlessness. ${ }^{2,5,6}$

In subjects with COPD, when psychological interventions for generalized anxiety and depression are used without exercise training or education, small effects (effect size of $-0.27,95 \% \mathrm{CI}-0.42$ to -0.14 ) that are not significantly different from controls are reported. ${ }^{5}$ In contrast, adding psychological interventions such as CBT to exercise training and education results in large and significant treatment effects for anxiety (effect size of -1.39 , $95 \%$ CI -2.19 to -0.59 ) and depression (effect size of $-0.86,95 \%$ CI -1.61 to -0.11$)^{2}$

Changes in the perceptual experience of dyspnea after repeated exposure to exercise training may result from improvements in system efficiency (less ventilatory effort for the same work load) and/or familiarity (reduced sensitivity to the sensation of breathlessness). ${ }^{7}$ Combining exercise training with strategies that target the cognitive processes underlying the perceptual experience of dyspnea may lead to greater improvements in health outcomes.

Pilot studies can be used to determine whether planning and resources a larger trial are warranted. In addition, these preliminary investigations provide practical information concerning the feasibility of planned recruitment strategy, intervention, and assessment protocols and provide an opportunity for preparatory training of study staff and development of data management processes. ${ }^{8,9}$

The aim of this pilot study was to develop and test a CBT program specific to the sensation of breathlessness. The specific objectives were to: (1) develop and implement a CBT program focused upon the sensation of breathlessness (Breathing: Recognize sensations, Explore thoughts and beliefs, Validate thoughts as useful or harmful, Evolve and change behavior [BREVE]) in a group of subjects concurrently participating in an 8-week compre-

\footnotetext{
This work was supported by seeding funds from the University of South Australia. Dr Williams has disclosed relationships with Boehringer Ingelheim and the Australian Physiotherapy Association. Dr Frith has disclosed relationships with Boehringer Ingelheim, Novartis, MediMark International, and the Lung Foundation Australia. Dr Paquet has disclosed no conflicts of interest.

This is Australian New Zealand Clinical Trials Registry trial ID ACTRN12614000505606.

Correspondence: Marie T Williams PhD, School of Population Health and Alliance for Research in Exercise, Nutrition and Activity (ARENA), School of Health Sciences, University of South Australia, City East Campus, North Terrace 5000, Adelaide, South Australia, Australia. E-mail: marie.williams@unisa.edu.au.
}

DOI: $10.4187 /$ respcare. 03764

\section{QUICK LOOK}

\section{Current knowledge}

Dyspnea is a multidimensional perceptual experience that reflects an individual's awareness of breathlessness intensity, sensory quality, and unpleasantness. Interventions that target the central processes for perception or cognition associated with dyspnea have been recognized as potential therapeutic strategies. To date, controlled trials of psychological interventions in subjects with COPD have targeted generalized anxiety, panic, and depression rather than focusing on the sensation of breathlessness.

\section{What this paper contributes to our knowledge}

A pilot cognitive behavioral therapy program was successfully implemented and positively received by the majority of subjects. The main practical issues identified included difficulty attaining a complete set of postintervention data and potential for group contamination. No significant differences were evident for descriptors of breathlessness or respiratory-related quality of life. There were significant improvements in the 6-min walk distance in the treatment group.

hensive pulmonary rehabilitation program, (2) identify any practical issues in the study protocol requiring review, and (3) estimate the beneficial effects of BREVE combined with the standard comprehensive pulmonary rehabilitation program compared with the standard program alone.

\section{Methods}

\section{Study Design}

This was a pilot study with a retrospective control.

\section{Subjects and Setting}

Consecutive patients with chronic respiratory conditions and associated breathing difficulties who intended to undertake the 8-week comprehensive pulmonary rehabilitation program at the Repatriation General Hospital in Adelaide, South Australia, were invited to participate in this study. They were excluded if they had cognitive or memory impairment (Mini Mental State Examination score $<23 / 30),{ }^{10}$ had clinically unstable respiratory or cardiac diseases or co-morbidities likely to render exercise unsafe (determined by the Repatriation General Hospital respiratory physician), or were currently registered for lung-volume-reduction surgery or lung transplantation. The control 
group comprised 58 subjects with COPD who had completed the same comprehensive pulmonary rehabilitation program in the years preceding the pilot study. ${ }^{7}$ Both groups (pilot and retrospective control) completed the same assessments. Ethical approval was granted by the human research ethics committees of the University of South Australia (P153/07) and the Repatriation General Hospital (P56/07). All subjects provided written informed consent.

\section{Sample Size}

Each individual cycle of the comprehensive pulmonary rehabilitation program ( 8 weeks) allowed a maximum of 25 subjects to participate. The BREVE program included a weekly psychologist-facilitated group session in which the maximum group size was set a priori at 12. All subjects referred and assessed for one comprehensive pulmonary rehabilitation program cycle were sequentially invited to participate in this pilot study.

\section{Measures}

Both the pilot study and control group subjects were scheduled to complete assessments within the 4 weeks preceding rehabilitation and again within 6 weeks of completing rehabilitation. Assessments and the comprehensive pulmonary rehabilitation program have been described previously. ${ }^{7}$ In summary, severity of airway obstruction was assessed by post-bronchodilator spirometry, confirmed by a respiratory specialist. Respiratory-related impairment was assessed using the modified Medical Research Council dyspnea scale. ${ }^{11}$ Arterial blood gas analysis was completed to determine resting-state hypoxemia. Exercise capacity was assessed using the better of duplicate 6-min walk tests as recommended in the American Thoracic Society guidelines. ${ }^{12}$ Disease-specific health-related quality of life was assessed with the St George Respiratory Questionnaire (SGRQ). ${ }^{13}$ Although there are a variety of instruments available for assessing dimensions of dyspnea, ${ }^{1}$ it has been recommended that choice of instrument or approach should align with the domain of dyspnea most relevant to the research objectives. ${ }^{1}$ The CBT intervention targeted the sensory-perceptual experience (predominantly sensory qualities and attendant cognitions and behaviors). The sensation of dyspnea was assessed at rest using a standardized protocol that records descriptors volunteered by subjects in their own words and selected from a preexisting list of breathlessness descriptors. ${ }^{1,14,15}$

\section{Intervention}

Each week of the standard 8-week comprehensive pulmonary rehabilitation program included 2 supervised exercise sessions (circuit training, only one supervised ses- sion in week 8) and $2 \mathrm{~h}$ of knowledge-based group education. Subjects were also encouraged to exercise daily at home (walking program). The total number of face-toface sessions in the standard program was 31 (16 education and 15 exercise sessions). The same health professionals (physiotherapist, psychologist, occupational therapist, respiratory nurse, and dietitian) provided these sessions for both groups (BREVE pilot and control).

The CBT program for the sensation of breathlessness (BREVE) was developed by a physiotherapist (MTW) and a health psychologist (PC) as a sequential series of 8 modules based on research concerning the neurophysiological and psychological mechanisms underpinning the sensation of dyspnea. ${ }^{1}$ The key principles of CBT include education based on cognitive restructuring, in which individuals identify and explore recurring thoughts (cognitions) to evaluate their accuracy (identify distortions and possibilities of catastrophizing) and practice coping strategies through progressive exposure to the stimulus. The BREVE program aimed to assist subjects to identify and describe their sensory experience of breathlessness (physical and affective components, worst vs daily), the thoughts and beliefs associated with these sensations, and their usual behavioral responses (eg, avoidance of or ending physical activity, specific environments) to learn whether the sensations of breathlessness posed an immediate valid threat to survival (beneficial vs catastrophizing cognitions) and the appropriateness of habitual behavioral responses (Table 1).

Each week, subjects attended a group session facilitated by a psychologist, supported by a program manual. Each week's module included a brief educational component (maximum 15-min presentation), discussion of a key concept, individual reflective activity, and explanation of homework tasks, including examples/demonstration and clarification of the task to be practiced during the supervised exercise sessions for the week. Subjects were encouraged to undertake homework each week by using cognitive strategies during the activities usually associated with breathlessness and fear at home. In addition to facilitating the group session, the psychologist also attended the supervised exercise sessions to coach individuals while breathless, in key tasks and techniques relevant to each module. The BREVE program intentionally did not replicate information included within the standard comprehensive pulmonary rehabilitation program educational sessions (respiratory anatomy, breathing exercises, pharmacologic management). The psychologist employed during the pilot study was experienced in CBT for the management of chronic pain, but had no prior experience of management of breathlessness, was naive to all outcome measures, and played no part in recruitment, outcome assessment, data management, or analysis.

Before or during the last session of the BREVE program, subjects were invited to complete a feedback form 
Table 1. Overview of the BREVE Program

\begin{tabular}{|c|c|c|c|c|}
\hline Module & Topic & Key Concepts & $\begin{array}{l}\text { Key Task Facilitated by Psychologist } \\
\text { in Group (1/wk) and Exercise (2/wk) } \\
\text { Sessions }\end{array}$ & $\begin{array}{l}\text { Key Task Practiced as } \\
\text { Homework }\end{array}$ \\
\hline 1 & $\begin{array}{l}\text { Recognize sensation of } \\
\text { breathlessness }\end{array}$ & $\begin{array}{l}\text { Dyspnea as a perceptual } \\
\text { process }\end{array}$ & Describe sensations of breathlessness & $\begin{array}{l}3 \text { activities that induce } \\
\text { dyspnea (3/wk) }\end{array}$ \\
\hline 2 & $\begin{array}{l}\text { Recognize thoughts/feelings } \\
\text { when breathless }\end{array}$ & Cognitions & Describe sensations and cognitions & $\begin{array}{l}3 \text { activities that induce } \\
\text { dyspnea (3/wk) }\end{array}$ \\
\hline 3 & $\begin{array}{l}\text { Do thoughts/feelings help or } \\
\text { hinder? }\end{array}$ & Helpful/harmful cognitions & $\begin{array}{l}\text { Describe sensation and identify } \\
\text { helpful/harmful cognitions }\end{array}$ & $\begin{array}{l}3 \text { activities that induce } \\
\text { dyspnea (3/wk) }\end{array}$ \\
\hline 4 & $\begin{array}{l}\text { Daily vs worst experience of } \\
\text { breathlessness }\end{array}$ & Memory and learning & $\begin{array}{l}\text { Compare usual with worst sensations } \\
\text { and cognitions }\end{array}$ & $\begin{array}{l}1 \text { activity usually avoided } \\
\text { due to dyspnea (3/wk) }\end{array}$ \\
\hline 5 & Breathlessness and behavior & $\begin{array}{l}\text { Anxiety/fear modify } \\
\text { behavior }\end{array}$ & $\begin{array}{l}\text { Identify habitual behaviors when } \\
\text { dyspneic }\end{array}$ & $\begin{array}{l}1 \text { activity usually avoided } \\
\text { due to dyspnea (3/wk) }\end{array}$ \\
\hline 6 & $\begin{array}{l}\text { Changing thoughts/feelings } \\
\text { when breathless }\end{array}$ & Distraction & Distraction techniques & $\begin{array}{l}30 \text { min of moderate intensity } \\
\text { exercise }(3 / \mathrm{wk})\end{array}$ \\
\hline 7 & $\begin{array}{l}\text { When to be concerned about } \\
\text { sensation of breathlessness }\end{array}$ & $\begin{array}{l}\text { Acute vs chronic } \\
\text { breathlessness }\end{array}$ & $\begin{array}{l}\text { Practice describing sensation and } \\
\text { likelihood of danger }\end{array}$ & $\begin{array}{l}30 \text { min of moderate intensity } \\
\text { exercise }(3 / \mathrm{wk})\end{array}$ \\
\hline 8 & $\begin{array}{l}\text { Exercise and BREVE as part } \\
\text { of life }\end{array}$ & $\begin{array}{l}\text { Review program, progress, } \\
\text { and feedback }\end{array}$ & Practice individual strategies & $\begin{array}{l}30 \text { min of moderate intensity } \\
\text { exercise }(3 / \mathrm{wk})\end{array}$ \\
\hline
\end{tabular}

BREVE = Breathing: Recognize sensations, Explore thoughts and beliefs, Validate thoughts as useful or harmful, Evolve and change behavior (intellectual property of the University of South Australia)

(12 items with a 4-point categorical scale: strongly agree, agree, disagree, strongly disagree) and provide comments on strengths and areas for improvement of the BREVE program. The intent of this feedback form was to collect key issues of the BREVE program content and usefulness. Many of the survey items were emotionally loaded in both a positive and negative sense to gain a clear impression of the perceived strengths and weaknesses of the program.

\section{Analyses}

Demographic characteristics of the pilot and retrospective control groups were collated and compared. The outcomes relevant for study objectives 1 (development of a CBT program specific for the sensation of breathlessness) and 2 (practical issues with study protocol) included feedback provided by pilot study subjects and sensation of breathlessness (no minimum clinically important difference available for descriptors). Responses were collated for the feedback form and analyzed descriptively for both categorical and opened-ended text-based items. Categorical options were collapsed into agree or disagree. Data for volunteered and endorsed descriptors of breathlessness were treated separately and allocated to groups of 6 categories for volunteered language and 4 categories for endorsed language. ${ }^{7}$ Descriptor data were treated as binary (subjects volunteered/endorsed or did not volunteer/endorse) within each descriptor category. The McNemar test was used to assess proportional changes within each descriptor category for the pilot and control groups (note that the McNemar test calculates proportional differences in the number of subjects that change categories [yes to no or no to yes] rather than the number of subjects who do not alter their responses). Due to the disparity in group sizes, between-group differences were assessed descriptively (percentage of subjects who changed or did not change descriptors within each category).

The CBT intervention aimed to alter the perception and cognitions associated with breathlessness. However, improving the experience of breathlessness without a subsequent improvement in functional exercise capacity or respiratory-related quality of life is unlikely to reduce health service usage. Therefore, the outcomes assessed to address study objective 3 (estimate of beneficial effects) were the 6-min walk distance (6MWD; minimum clinically important difference of $25 \mathrm{~m})^{16}$ and SGRQ total score (minimum clinically important difference of 4 units). ${ }^{17}$

All analyses were undertaken using SAS 9.2 (SAS Institute, Cary, North Carolina), with $P<.05$ regarded as statistically significant. Missing post-intervention data were imputed by predicted values derived from linear regression models based on pre-intervention values. Repeatedmeasures analysis of variance was used to assess withingroup intervention effects (pre to post) and differences in intervention effects between groups (BREVE pilot or control).

The effect size is a way of quantifying the effect of the intervention in both groups rather than the statistical significance (which includes both effect and sample sizes). ${ }^{18}$ Effect sizes were calculated post hoc ${ }^{18}$ with Cohen's d, 
Table 2. Baseline Characteristics for Pilot (BREVE + Comprehensive Pulmonary Rehabilitation Program) and Control (Comprehensive Pulmonary Rehabilitation Program Only) Groups

\begin{tabular}{|c|c|c|}
\hline Baseline Characteristic & $\begin{array}{l}\text { Pilot Group } \\
\quad(n=12)\end{array}$ & $\begin{array}{l}\text { Control Group } \\
\quad(n=58)\end{array}$ \\
\hline Age, y & $75 \pm 6$ & $71 \pm 9$ \\
\hline Males/females, $n$ & $11 / 1$ & $36 / 22$ \\
\hline BMI, $\mathrm{kg} / \mathrm{m}^{2}$ & $28 \pm 7$ & $27 \pm 5$ \\
\hline $\mathrm{FEV}_{1} / \mathrm{FVC}$ & $0.54 \pm 0.09$ & $0.46 \pm 0.15$ \\
\hline $\mathrm{FEV}_{1}, \%$ predicted & $53 \pm 29$ & $58 \pm 24$ \\
\hline $\mathrm{P}_{\mathrm{aO}_{2}}, \mathrm{~mm} \mathrm{Hg}$ & $70.5 \pm 9.0$ & $72.8 \pm 9.0$ \\
\hline $\mathrm{P}_{\mathrm{aCO}_{2}}, \mathrm{~mm} \mathrm{Hg}$ & $39.8 \pm 3.8$ & $39.8 \pm 5.3$ \\
\hline $\begin{array}{l}\text { Modified Medical Research } \\
\text { Council score }\end{array}$ & $2 \pm 1$ & $2 \pm 1$ \\
\hline 6MWD, m & $375 \pm 125$ & $384 \pm 109$ \\
\hline SGRQ, total score & $39 \pm 6$ & $48 \pm 15$ \\
\hline $\begin{array}{l}\text { Attendance at exercise/education } \\
\text { sessions }(\max =31), \%\end{array}$ & $88 \pm 9$ & $81 \pm 21$ \\
\hline \multicolumn{3}{|c|}{$\begin{array}{l}\text { Values are expressed as mean } \pm \text { SD except where indicated. } \\
\text { BREVE }=\text { Breathing: Recognize sensations, Explore thoughts and beliefs, Validate thoughts } \\
\text { as useful or harmful, Evolve and change behavior } \\
\text { BMI }=\text { body mass index } \\
6 \mathrm{MWD}=6 \text {-min walk distance } \\
\text { SGRQ }=\text { St George Respiratory Questionnaire }\end{array}$} \\
\hline
\end{tabular}

where effect sizes were classified as negligible $(\geq-0.15$ and $<0.15$ ), small ( $\geq 0.15$ and $<0.40$ ), medium $(\geq 0.40$ and $<0.75)$, large $(\geq 0.75$ and $<1.10)$, very large $(\geq 1.10$ and $<1.45)$, and huge $(\geq 1.45) .{ }^{19}$

\section{Results}

Each of the first 12 consecutively recruited subjects agreed to participate in this pilot study, with 11 completing the intervention (comprehensive pulmonary rehabilitation program + BREVE) and follow-up assessments. The majority of pilot group subjects met the spirometric criteria for COPD $(9 / 11,82 \%$; GOLD [Global Initiative for Chronic Obstructive Lung Disease] stage $2=2$, GOLD stage $3=5$, GOLD stage $4=2$ ). ${ }^{20}$ With the exception of the ratio of males to females in each group, there were no significance differences between characteristics of the pilot group compared with the control group (Table 2). Missing data for primary outcomes necessitated imputation by linear regression (post-intervention 6MWD $n=6$, SGRQ score $n=3)$.

\section{Feedback Concerning the BREVE Program}

Six of the 11 subjects provided written feedback: 5 used the feedback form, although not all respondents completed each item, and one provided handwritten feedback without the feedback form (Table 3). Subjects unanimously sup- ported the need for a group session and the role of a facilitator in both the group session and the supervised exercise sessions. The majority of subjects agreed that the program was useful in helping them understand and manage their breathlessness and confirmed the layout and language style of the BREVE manual. Areas of revision/improvement included reducing and altering the homework tasks.

\section{Volunteered and Endorsed Descriptors of Breathlessness}

Within Groups. Within the pilot group, the proportion of subjects who changed descriptors of breathlessness preto post-intervention was not significant (volunteered or endorsed) (Table 4), whereas within the control group, a significant proportion of subjects reduced their use of volunteered descriptors in 2 volunteered language categories (air hunger $[P=.03]$ and depressed, regret, helpless $[P=.04]$; detailed tables are provided by Williams et $\left.\mathrm{al}^{7}\right)$.

Between Groups. For both the pilot and control groups, the percentage of subjects changing or not changing descriptors within each language category was similar (Table 5).

\section{MWD and SGRQ Score}

Within Groups. For both the pilot $(n=11)$ and control $(n=59)$ groups, there were clinically relevant improvements in the 6MWD after the intervention (Table 6). Mean differences for the SGRQ (total score) were small and did not reach statistical or clinical importance (mean change in points of 2) in either the pilot or control group.

Between Groups. The mean increase in the 6MWD was greater for the pilot group compared with the control group $(P=.03$, effect size of 0.69$)$. No significant difference was evident for the SGRQ (total score) between groups $(P=.93$, effect size of 0.03) (see Table 6).

\section{Discussion}

The primary objectives of this pilot study were to develop and implement a CBT program specifically targeting the sensation of breathlessness (BREVE), identify practical issues with the study protocol, and estimate any additional benefit of the CBT program to standard comprehensive pulmonary rehabilitation. The CBT program was successfully implemented and positively received by the majority of subjects. The main practical issues identified throughout the pilot study included difficulty attaining a complete set of post-intervention data, potential for group contamination, and choice of outcome measures. No sig- 
Table 3. Summary of Subjects' Feedback on the BREVE Program

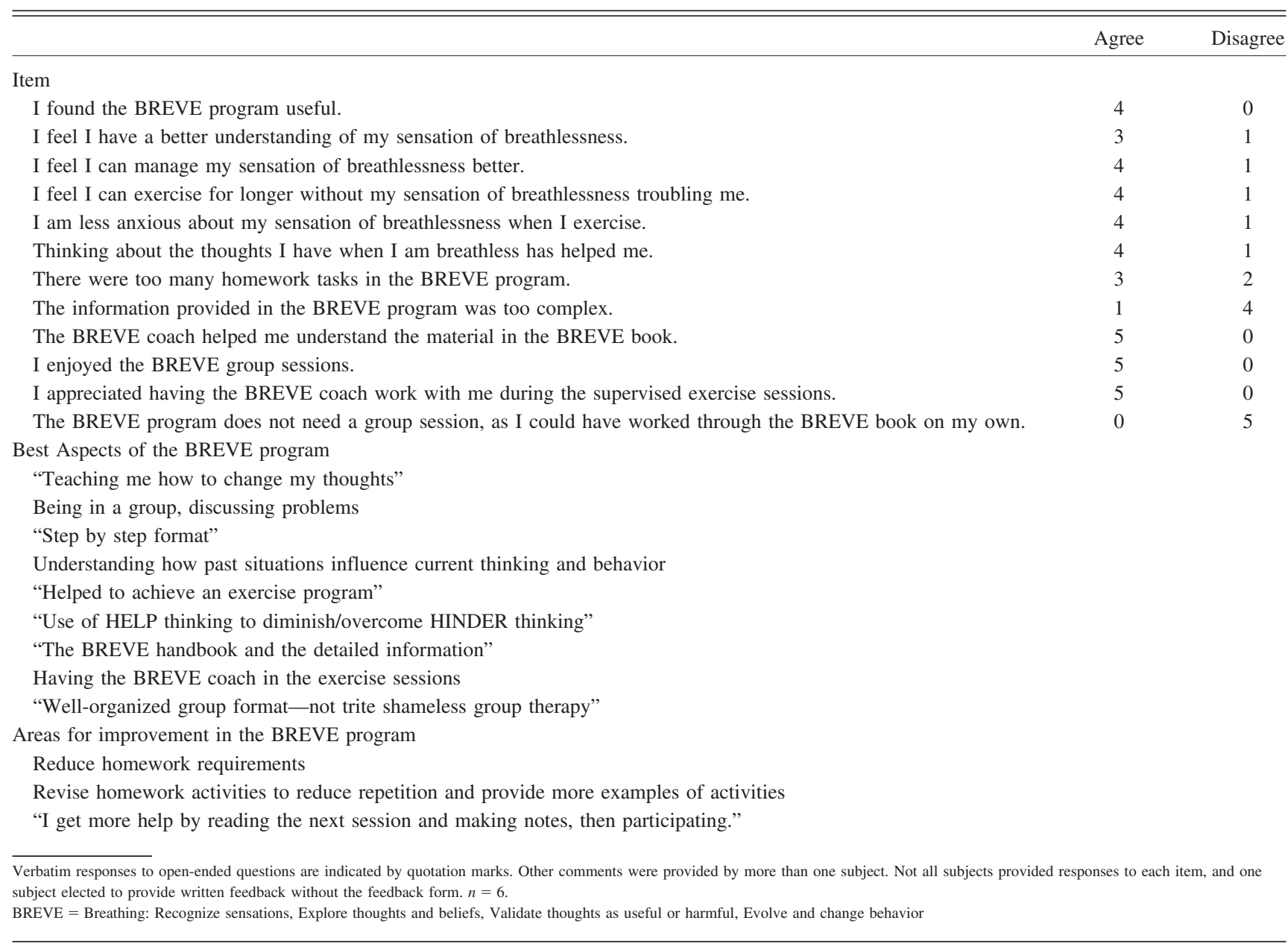

nificant differences were evident for descriptors of breathlessness (volunteered and endorsed) or respiratory-related quality of life within or between the pilot and control groups. There were significant improvements in the 6MWD within and between pilot and control groups, with greater mean improvement for the pilot group (mean of $57 \mathrm{~m}$ vs $27 \mathrm{~m})$.

In theory, altering the way the sensation of dyspnea is perceived, especially reducing the fear/threat response, should alter behavioral outcomes. The model of Lansing et al ${ }^{21}$ for the perception of dyspnea proposed a sequence in which initially somatosensory areas assess the intensity and sensory quality (descriptors) of the breathlessness (somatic awareness), with a subsequent stage managed by the insula, dorsal anterior cingulate cortex, amygdala, and medial thalamus assessing the degree of unpleasantness/threat (affective awareness). ${ }^{22}$ Where the degree of unpleasantness is perceived to be threatening, immediate and longerterm behavioral responses are learned (such as early cessation of activity or avoidance). Both stages may be modulated by cognitive processes (memory, learning, and schemata) and personality (affectivity). ${ }^{3}$

The findings of this pilot study appear to be consistent with this model if the feedback provided by BREVE program subjects (increased endurance, better breathlessness management, and reduced anxiety associated with exercise-related breathlessness) reflects a change in affective awareness facilitating an ability to increase their exercise capacity. A heavy emphasis on cognitive restructuring and repeated exposure to potentially aversive sensations (in this case, breathlessness) can be an effective way of reducing the fear associated with physical sensations, ${ }^{23}$ may facilitate more effective exercise training, and potentially could lead to larger or longer-lasting changes in activity levels. Previous studies have noted the relationship between levels of anxiety sensitivity and breathlessness. ${ }^{23}$ Anxiety and/or anxiety sensitivity may have been moderated with the BREVE intervention, with reduced anxiety associated with exercise-induced breathlessness reported by BREVE subjects. A formal assessment of anxiety and 
Table 4. Number of Subjects Who Endorsed/Volunteered Language Category Descriptors Before and After Completion of the Comprehensive Pulmonary Rehabilitation and BREVE Programs

\begin{tabular}{|c|c|c|c|c|}
\hline \multirow{3}{*}{ Language Category } & \multirow{2}{*}{\multicolumn{3}{|c|}{$\begin{array}{l}\text { Subjects Who Endorsed/Volunteered } \\
\text { Descriptors }(n)\end{array}$}} & \multirow{3}{*}{$\begin{array}{l}P \text { (Odds Ratio, } \\
95 \% \text { CI)* }\end{array}$} \\
\hline & & & & \\
\hline & No & Yes & Total & \\
\hline \multicolumn{5}{|l|}{ Volunteered language categories } \\
\hline \multicolumn{5}{|l|}{ Air hunger } \\
\hline \multicolumn{5}{|c|}{ Before standard program + BREVE } \\
\hline No & 6 & 2 & 8 & \multirow[t]{3}{*}{$.62(1.00,0.07-13.79)$} \\
\hline Yes & 2 & 1 & 3 & \\
\hline Total & 8 & 3 & 11 & \\
\hline \multicolumn{5}{|l|}{ Work } \\
\hline \multicolumn{5}{|c|}{ Before standard program + BREVE } \\
\hline No & 8 & 1 & 9 & \multirow[t]{3}{*}{$.48(1.00,0.01-78.49)$} \\
\hline Yes & 1 & 1 & 2 & \\
\hline Total & 9 & 2 & 11 & \\
\hline \multicolumn{5}{|l|}{ Tight } \\
\hline \multicolumn{5}{|c|}{ Before standard program + BREVE } \\
\hline No & 10 & 0 & 10 & \multirow{3}{*}{$>.99(0.0,0.0-39.0)$} \\
\hline Yes & 1 & 0 & 1 & \\
\hline Total & 11 & 0 & 11 & \\
\hline \multicolumn{5}{|l|}{ Frightening, awful, worried } \\
\hline \multicolumn{5}{|c|}{ Before standard program + BREVE } \\
\hline No & 6 & 0 & 6 & \multirow[t]{3}{*}{$.13(0.0,0.0-1.51)$} \\
\hline Yes & 4 & 1 & 5 & \\
\hline Total & 10 & 1 & 11 & \\
\hline \multicolumn{5}{|l|}{ Uncomfortable, annoying } \\
\hline \multicolumn{5}{|c|}{ Before standard program + BREVE } \\
\hline No & 2 & 1 & 3 & \multirow[t]{3}{*}{$.37(0.25,0.01-2.53)$} \\
\hline Yes & 4 & 4 & 8 & \\
\hline Total & 6 & 5 & 11 & \\
\hline \multicolumn{5}{|l|}{ Depressed, regretful, helpless } \\
\hline \multicolumn{5}{|c|}{ Before standard program + BREVE } \\
\hline No & 9 & 0 & 9 & $.48(0.0,0.0-5.32)$ \\
\hline Yes & 2 & 0 & 2 & \\
\hline Total & 11 & 0 & 11 & \\
\hline Endorsed language categories & & & & \\
\hline Air hunger & & & & \\
\hline Before standard program + & & & & \\
\hline No & 4 & 0 & 4 & NA \\
\hline Yes & 0 & 7 & 7 & \\
\hline Total & 4 & 7 & 11 & \\
\hline Work/effort & & & & \\
\hline Before standard program + & & & & \\
\hline No & 3 & 3 & 6 & $.62(3.00,0.24-157.49)$ \\
\hline Yes & 1 & 4 & 5 & \\
\hline Total & 4 & 7 & 11 & \\
\hline Tight & & & & \\
\hline Before standard program + & & & & \\
\hline No & 9 & 0 & 9 & $.48(0.0,0.0-5.32)$ \\
\hline Yes & 2 & 0 & 2 & \\
\hline Total & 11 & 0 & 11 & \\
\hline & & & & (continued) \\
\hline
\end{tabular}


Table 4. Continued

\begin{tabular}{|c|c|c|c|c|}
\hline \multirow{3}{*}{ Language Category } & \multirow{2}{*}{\multicolumn{3}{|c|}{$\begin{array}{l}\text { Subjects Who Endorsed/Volunteered } \\
\text { Descriptors }(n)\end{array}$}} & \multirow{3}{*}{$\begin{array}{l}P(\text { Odds Ratio, } \\
95 \% \mathrm{CI})^{*}\end{array}$} \\
\hline & & & & \\
\hline & No & Yes & Total & \\
\hline \multicolumn{5}{|l|}{ Unnamed } \\
\hline \multicolumn{5}{|c|}{ Before standard program + BREVE } \\
\hline No & 2 & 1 & 3 & \multirow{3}{*}{$>.99(0.50,0.01-9.60)$} \\
\hline Yes & 2 & 6 & 8 & \\
\hline Total & 4 & 7 & 11 & \\
\hline \multicolumn{5}{|c|}{$\begin{array}{l}\text { * McNemar test } \\
\text { BREVE = Breathing: Recognize sensations, Explore thoughts and beliefs, Validate thoughts as useful or harmful, Evolve and change behavior } \\
\text { Standard program = comprehensive pulmonary rehabilitation program } \\
\text { NA = unable to calculate because one or more cells contained an insufficient number of subjects }\end{array}$} \\
\hline
\end{tabular}

Table 5. Proportion of Pilot and Control Group Subjects Who Changed Pre- to Post-Intervention Descriptors

\begin{tabular}{|c|c|c|c|c|}
\hline \multirow[t]{2}{*}{ Language Category } & \multicolumn{2}{|c|}{$\begin{array}{l}\text { No Change } \\
\text { (Pre- to Post- } \\
\text { Intervention), \% }\end{array}$} & \multicolumn{2}{|c|}{$\begin{array}{c}\text { Change (Pre- } \\
\text { to Post- } \\
\text { Intervention), \% }\end{array}$} \\
\hline & Pilot & Control & Pilot & Control \\
\hline \multicolumn{5}{|l|}{$\begin{array}{l}\text { Volunteered language } \\
\text { category }\end{array}$} \\
\hline Air hunger & 64 & 64 & 36 & 36 \\
\hline Work & 82 & 81 & 18 & 19 \\
\hline Tight & 91 & 86 & 9 & 14 \\
\hline $\begin{array}{l}\text { Frightening, awful, } \\
\text { worried }\end{array}$ & 64 & 66 & 36 & 34 \\
\hline Uncomfortable, annoying & 55 & 64 & 45 & 36 \\
\hline $\begin{array}{l}\text { Depressed, regretful, } \\
\text { helpless }\end{array}$ & 82 & 67 & 18 & 33 \\
\hline Mean & 73 & 71 & 27 & 29 \\
\hline \multicolumn{5}{|l|}{ Endorsed language category } \\
\hline Air hunger & 100 & 66 & 0 & 34 \\
\hline Work/effort & 64 & 64 & 36 & 36 \\
\hline Tight & 82 & 66 & 18 & 34 \\
\hline Unnamed & 73 & 72 & 27 & 28 \\
\hline Mean & 80 & 67 & 20 & 33 \\
\hline
\end{tabular}

depression should be included in any future randomized controlled trial.

The proportion of subjects in the pilot group who changed descriptors of breathlessness between baseline and postintervention assessment was not statistically significant (see Table 4), most likely due to the very small sample size. On average, 3 subjects (27\%) changed their choice of descriptors within each of the volunteered or endorsed descriptor categories, with the largest change in the frightening and uncomfortable categories (both $n=4,36 \%$ ). The percentage of subjects who changed descriptors of breathlessness strongly associated with greater unpleasantness (air hunger, frightening, uncomfortable) may be more important than whether the proportional change reached statistical significance. The percentage of subjects who changed or did not change descriptors of breathlessness after the intervention followed similar patterns in the pilot and control groups (see Table 5). Using a binary method of assessment (a subject either volunteered or endorsed a descriptor within a category or did not), we assessed whether subjects acknowledged the presence of the descriptor rather than the intensity of that sensation. More recent instruments, such as Dyspnea-1224 and the Multidimensional Dyspnea Profile, ${ }^{25}$ permit the assessment of both the presence and intensity of the descriptor.

Although subjects' feedback was positive for the BREVE program and there appeared to be a greater improvement in exercise outcomes, there were a number of confounding issues within the pilot study (eg, disparity between group sizes, use of a retrospective control group), with one of the main issues being the amount of missing data for primary outcomes post-intervention (6MWD $n=6$, SGRQ score $n=3$ ) due to the reduced number of staff and staff changes, resulting in delayed, rescheduled, and cancelled appointments. Exacerbations of chronic disease, lack of time, transport issues, motivation, and lack of perceived benefit of pulmonary rehabilitation are well documented as reasons for withdrawal or dropout in studies of pulmonary rehabilitation. ${ }^{26,27}$ If the incomplete post-intervention data are considered to reflect the overall dropout rate for this pilot study, then the range for different outcomes (6MWD, SGRQ score) falls within previously reported estimates for dropout/withdrawal rates of subjects in pulmonary rehabilitation programs $(0-54 \%) .{ }^{26}$ In planning a larger trial, a contingency plan ensuring against unnecessary dropout and loss to follow-up is warranted (dedicated and funded study staff, prescheduled days booked for assessments of study subjects, limiting the number of rescheduled appointments [excluding subject-requested alterations]). 
Table 6. Within- and Between-Group Analyses for 6MWD and SGRQ

\begin{tabular}{|c|c|c|c|c|c|}
\hline \multirow[b]{2}{*}{ Outcome Measure and Group } & \multirow[b]{2}{*}{ Assessment } & \multirow[b]{2}{*}{ Mean \pm SD } & \multirow[b]{2}{*}{ Mean Difference \pm SD } & \multicolumn{2}{|c|}{$P$ (Effect Size, Cohen's d) } \\
\hline & & & & $\begin{array}{l}\text { Within } \\
\text { Groups }\end{array}$ & $\begin{array}{c}\text { Between } \\
\text { Groups }\end{array}$ \\
\hline \multicolumn{6}{|l|}{$6 \mathrm{MWD}, \mathrm{m}$} \\
\hline Pilot & Pre & $375.09 \pm 124.56$ & $57.4 \pm 44.62$ & $.005(-0.73)$ & $.03 *(-0.69)$ \\
\hline Standard program + BREVE & Post & $453.0 \pm 43.90$ & & & \\
\hline Control & Pre & $383.65 \pm 109.27$ & $26.6 \pm 55.65$ & $.006(0.23)$ & \\
\hline Standard program & Post & $410.22 \pm 119.12$ & & & \\
\hline \multicolumn{6}{|l|}{ SGRQ, total score } \\
\hline Pilot & Pre & $39.17 \pm 14.76$ & $-2.3 \pm 9.11$ & $.42(0.15)$ & $.93(0.03)$ \\
\hline Standard program + BREVE & Post & $37.73 \pm 19.35$ & & & \\
\hline Control & Pre & $46.76 \pm 16.22$ & $-2.1 \pm 11.83$ & $.18(0.12)$ & \\
\hline Standard program & Post & $44.67 \pm 17.73$ & & & \\
\hline $\begin{array}{l}* P<.05 \text { (mean } \pm \mathrm{SD} \text { are arithmetic from } \\
6 \mathrm{MWD}=6 \text {-min walk distance } \\
\mathrm{SGRQ}=\mathrm{St} \text { George Respiratory Questionn } \\
\text { Standard program }=\text { comprehensive pulmo } \\
\text { BREVE = Breathing: Recognize sensation }\end{array}$ & $\begin{array}{l}\text { ilitation program } \\
\text { thoughts and beli }\end{array}$ & thoughts as useful or & Evolve and change behavior & & \\
\hline
\end{tabular}

The first 12 consecutive subjects agreed to participate in this pilot study, and only one subject did not complete the intervention. This uptake rate is better than expected but falls within reported participation rates (range of 35-100\%) for previous studies of non-pharmacologic interventions for subjects with COPD. ${ }^{28}$ This positive responder bias may have been a result of recruitment by the comprehensive pulmonary rehabilitation program director, novelty of the intervention, or scheduling the intervention within the standard program (no additional travel or appointments were required) or due to the previous commitment of the subjects to attend the comprehensive pulmonary rehabilitation program and their being predisposed to changing health behaviors. One unanticipated issue that arose midway during the program was the request from a subject undertaking the standard comprehensive pulmonary rehabilitation program to attend the BREVE group sessions (which was accepted). Pilot group subjects completed the standard program with the entire cohort scheduled within that rehabilitation cycle, which permitted discussion and conversation concerning the nature of the extra group BREVE session and the presence of the facilitator during the supervised exercise sessions. In planning a larger trial, block randomization (comprehensive pulmonary rehabilitation program cycles) rather than individual randomization will be required to reduce the potential for crosscontamination between trial groups.

A number of authors recommend against the use of inferential statistics, or at least a reduced emphasis on statistical significance, in pilot studies that include small samples.9,29 Although the imputation process may have inflated the mean change for the 6MWD, the available raw data for the pilot group's 6MWD pre- to post-intervention
$(57 \pm 45 \mathrm{~m})$ was greater than the mean difference for the control group (27 $\pm 56 \mathrm{~m})$ and above the minimum clinically important difference suggested for the 6MWD. 16,30-32

The 6-min walk test and SGRQ have been previously used to assess responsiveness to pulmonary rehabilitation. In the pilot and control groups, the mean increase in 6MWD (both raw and imputed) exceeded the 25-m improvement (95\% CI 20-61 m) suggested by Holland et al, ${ }^{16}$ but only the pilot group surpassed all other estimates for the minimum clinically important difference, ${ }^{30,31}$ with a value of $54 \mathrm{~m}(95 \%$ CI $37-71 \mathrm{~m}) .{ }^{32}$ However, neither the pilot nor control group achieved statistically significant improvements in quality of life at follow-up, and no significant difference was evident for the SGRQ (total score) between groups ( $P=.16$, mean change of 2 points) (see Table 3 ). Although neither group achieved the minimum clinically important difference of 4 points, both achieved the lower range of changes considered clinically meaningful (change between 2 and 8 points $^{33}$ ), and there was no difference in these changes between historical control and pilot groups.

There may be several reasons why neither group demonstrated significant improvements in respiratory-related quality of life despite both groups having improvements in functional exercise assessments. The most likely reasons include the appropriateness of the SGRQ for the study population, the lack of a social group control for the CBT intervention, and the novelty/priority given to the exercise component of the BREVE program.

The SGRQ was originally developed for asthma and COPD, but, in the clinical research environment, is used predominantly in COPD populations. A review of the SGRQ undertaken to create a more appropriate instrument for use in patients with COPD identified 26 items that 
required modification. ${ }^{34}$ At the time of this pilot study, the SGRQ was the recommended instrument for assessing quality of life for patients undertaking pulmonary rehabilitation within the Australian context, and the revised version of the SGRQ was not yet available.

The pilot and control groups completed a similar pulmonary rehabilitation program (including the staff and the number of supervised exercise sessions attended). The greater improvement in exercise capacity in the pilot group might be due to the additional CBT group session each week and/or individualized coaching of cognitive strategies by the psychologist addressing the perception of breathlessness during the supervised exercise sessions. It is possible that the nature of these group sessions created factors known to improve performance, such as individualized attention and interactive sessions involving the sharing and addressing of significant fears, as well as the novel attraction of the intervention contributing to increased group cohesion, a variable often linked to positive group exercise performance. ${ }^{35}$ Additionally, social support has been linked with improved health outcomes, including better adherence and increased exercise tolerance among the elderly and cardiac rehabilitation populations. ${ }^{36,37}$ Furthermore, the overt evaluation embedded in regular observation and homework for the pilot group members may have led them to work harder in the gym, commonly known as social facilitation. ${ }^{38}$ However, the longer-term maintenance of these benefits has not been assessed.

By their nature, pilot studies commonly include small sample sizes (underpowered) and a variety of known and unforeseen methodological limitations. For these reasons, estimating sample sizes for larger trials from pilot study results, although common practice, is contentious. ${ }^{8}$ Within this pilot study, the effect sizes calculated for the primary outcomes differed substantially (6MWD medium, SGRQ score negligible). Therefore, to estimate the sample size required for a larger randomized control trial with follow-up assessments, a conservative approach was applied. Using 6MWD as the primary outcome, the minimum sample size required for a small effect size $(0.15)$, if $\mathrm{P}=.05$ and power is $80 \%$, for 2 time points (baseline and 1 month post-intervention) is 90 (45 subjects/group), for 3 time points (baseline and 1 and 6 months post-intervention) is 74 (37 subjects/group), and for 4 time points (baseline and 1,6 , and 12 months post-intervention) is 62 (31 subjects/group).

\section{Conclusions}

This pilot study developed a CBT intervention specific to the sensation of breathlessness that appears feasible and acceptable to subjects undertaking pulmonary rehabilitation. Estimates of beneficial effects were limited by the missing post-intervention data, and a number of practical issues were identified with the protocol. These include securing dedicated project staff and transport, adding a social group control, block rather than individual randomization, minor alterations to the BREVE program, revising the outcome measures, and extending the follow-up period to assess longevity of benefits. These modifications have been made to a larger randomized control trial currently under way (Australian New Zealand Clinical Trials Registry trial ID ACTRN12611000292976, supported by National Health and Medical Research Council project grant 1010309).

\section{ACKNOWLEDGMENTS}

We thank Anne Southwell (Pulmonary Rehabilitation, Repatriation General Hospital) for her diligent and outstanding management of subjects and data. We thank Mr John Petkov, our friend and dedicated mathematician, for his generous and enthusiastic critique and analysis. John's untimely death leaves us poorer as people and researchers.

\section{REFERENCES}

1. Parshall MB, Schwartzstein RM, Adams L, Banzett RB, Manning HL, Bourbeau J, et al. An official American Thoracic Society statement: update on the mechanisms, assessment, and management of dyspnea. Am J Respir Crit Care Med 2012;185(4):435-452.

2. Coventry PA, Gellatly JL. Improving outcomes for COPD patients with mild-to-moderate anxiety and depression: a systematic review of cognitive behavioural therapy. Br J Health Psychol 2008;13 (Pt 3):381-400.

3. von Leupoldt A, Dahme B. Psychological aspects in the perception of dyspnea in obstructive pulmonary diseases. Respir Med 2007; 101(3):411-422.

4. von Leupoldt A, Fritzsche A, Trueba AF, Meuret AE, Ritz T. Behavioral medicine approaches to chronic obstructive pulmonary disease. Ann Behav Med 2012;44(1):52-65.

5. Baraniak A, Sheffield D. The efficacy of psychologically based interventions to improve anxiety, depression and quality of life in COPD: a systematic review and meta-analysis. Patient Educ Couns 2011;83(1):29-36.

6. Wiles L, Cafarella P, Williams M. Exercise training combined with psychological interventions for people with chronic obstructive pulmonary disease. Respirology 2015;20(1):46-55.

7. Williams MT, Petkov J, Olds TS, Cafarella P, Frith P. A reduction in the use of volunteered descriptors of air hunger is associated with increased walking distance in people with COPD. Respir Care 2012; 57(9):1431-1441.

8. Kraemer HC, Mintz J, Noda A, Tinklenberg J, Yesavage JA. Caution regarding the use of pilot studies to guide power calculations for study proposals. Arch Gen Psychiat 2006;63(5):484-489.

9. Fain JA. Should we publish pilot/feasibility studies? Diabetes Educ 2010;36(4):521-521.

10. Folstein MF, Folstein SE, McHugh PR. "Mini-mental state": a practical method for grading the cognitive state of patients for the clinician. J Psychiatr Res 1975;12(3):189-198.

11. American Thoracic Society. Surveillance for respiratory hazards in the occupational setting. Am Rev Respir Dis 1982;126(5):952-956.

12. ATS Committee on Proficiency Standards for Clinical Pulmonary Function Laboratories. ATS statement: guidelines for the six-minute walk test. Am J Respir Crit Care Med 2002;166(1):111-117.

13. Jones PW, Quirk FH, Baveystock CM, Littlejohns P. A self-complete measure of health status for chronic airflow limitation: the St. 


\section{Cognitive Behavioral Therapy for Management of Dyspnea}

George's Respiratory Questionnaire. Am Rev Respir Dis 1992;145(6): 1321-1327.

14. Mahler DA, Harver A, Lentine T, Scott JA, Beck K, Schwartzstein RM. Descriptors of breathlessness in cardiorespiratory diseases. Am J Respir Crit Care Med 1996;154(5):1357-1363.

15. Williams M, Cafarella P, Olds T, Petkov J, Frith P. Affective descriptors of the sensation of breathlessness are more highly associated with severity of impairment than physical descriptors in people with COPD. Chest 2010;138(2):315-322.

16. Holland AE, Hill CJ, Rasekaba T, Lee A, Naughton MT, McDonald CF. Updating the minimal important difference for six-minute walk distance in patients with chronic obstructive pulmonary disease. Arch Phys Med Rehabil 2010;91(2):221-225.

17. Jones P. Interpreting thresholds for a clinically significant change in health status in asthma and COPD. Eur Respir J 2002;19(3):398-404.

18. Coe R. It's the effect size, stupid. What effect size is and why it is important. Paper presented at the British Educational Research Association annual conference, Exeter, 12-14 September, 2002. http:// www.cem.org/attachments/ebe/ESguide.pdf. Accessed April 4, 2015.

19. Thalheimer W, Cook S. How to calculate effect sizes from published research: a simplified methodology. http://www.bwgriffin.com/gsu/ courses/edur9131/content/Effect_Sizes_pdf5.pdf. Accessed April 4, 2015.

20. Global Initiative for Chronic Obstructive Lung Disease. Global strategy for the diagnosis, management, and prevention of chronic obstructive pulmonary disease. 2011. http://www.goldcopd.org/uploads/ users/files/GOLD_Report_2011_Feb21.pdf. Accessed April 3, 2015.

21. Lansing RW, Gracely RH, Banzett RB. The multiple dimensions of dyspnea: review and hypotheses. Respir Physiol Neurobiol 2009; 167(1):53-60

22. von Leupoldt A, Sommer T, Kegat S, Baumann HJ, Klose H, Dahme B, Büchel C. Dyspnea and pain share emotion-related brain network. Neuroimage 2009;48(1):200-206.

23. Simon NM, Weiss AM, Kradin R, Evans KC, Reese HE, Otto MW, et al. The relationship of anxiety disorders, anxiety sensitivity and pulmonary dysfunction with dyspnea-related distress and avoidance. J Nerv Ment Dis 2006;194(12):951-957.

24. Yorke J, Moosavi SH, Shuldham C, Jones PW. Quantification of dyspnoea using descriptors: development and initial testing of the Dyspnoea-12. Thorax 2010;65(1):21-26.

25. Meek PM, Banzett R, Parshall MB, Gracely RH, Schwartzstein RM, Lansing R. Reliability and validity of the multidimensional dyspnea profile. Chest 2012;141(6):1546-1553.
26. Bjoernshave B, Korsgaard J, Nielsen CV. Does pulmonary rehabilitation work in clinical practice? A review on selection and dropout in randomized controlled trials on pulmonary rehabilitation. Clin Epidemiol 2010;2:73-83.

27. Keating A, Lee AL, Holland AE. Lack of perceived benefit and inadequate transport influence uptake and completion of pulmonary rehabilitation in people with chronic obstructive pulmonary disease: a qualitative study. J Physiother 2011;57(3):183-190.

28. Sohanpal R, Hooper R, Hames R, Priebe S, Taylor S. Reporting participation rates in studies of non-pharmacological interventions for patients with chronic obstructive pulmonary disease: a systematic review. Syst Rev 2012;1:66.

29. Thabane L, Ma J, Chu R, Cheng J, Ismaila A, Rios LP, et al. A tutorial on pilot studies: the what, why and how. BMC Med Res Methodol 2010;10:1.

30. Lacasse Y, Goldstein R, Lasserson T, Martin S. Pulmonary rehabilitation for chronic obstructive pulmonary disease. Cochrane Database Syst Rev 2006;4:CD003793.

31. Puhan MA, Mador MJ, Held U, Goldstein R, Guyatt GH, Schünemann HJ. Interpretation of treatment changes in 6-minute walk distance in patients with COPD. Eur Respir J 2008;32(3):637-643.

32. Redelmeier DA, Bayoumi AM, Goldstein RS, Guyatt GH. Interpreting small differences in functional status: the six minute walk test in chronic lung disease patients. Am J Respir Crit Care Med 1997; 155(4):1278-1282.

33. Glaab T, Vogelmeier C, Buhl R. Outcome measures in chronic obstructive pulmonary disease (COPD): strengths and limitations. Respir Res 2010;11:79.

34. Meguro M, Barley EA, Spencer S, Jones PW. Development and validation of an improved, COPD-specific version of the St. George respiratory questionnaire. Chest 2007;132(2):456-463.

35. Dunlop WL, Falk CF, Beauchamp MR. How dynamic are exercise group dynamics? Examining changes in cohesion within class-based exercise programs. Health Psychol 2013;32(12):1240-1243.

36. Fraser SN, Rodgers WM, Murray TC, Daub B. The enduring impact of social factors on exercise tolerance in men attending cardiac rehabilitation. J Cardiopulm Rehabil Prev 2007;27(2):92-96.

37. Karelina K, DeVries AC. Modeling social influences on human health. Psychosom Med 2011;73(1):67-74.

38. Anderson-Hanley C, Snyder AL, Nimon JP, Arciero PJ. Social facilitation in virtual reality-enhanced exercise: competitiveness moderates exercise effort of older adults. Clin Interv Aging 2011;6:275280. 